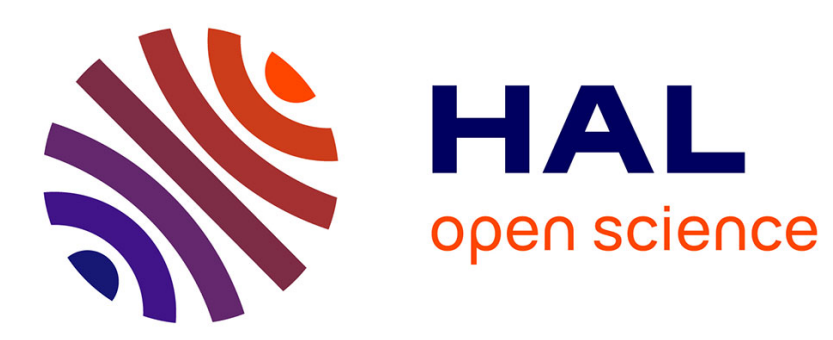

\title{
Crystallization of polypropylene at high cooling rates
}

\author{
Séverine A.E. Boyer, Jean-Marc Haudin
}

\section{To cite this version:}

Séverine A.E. Boyer, Jean-Marc Haudin. Crystallization of polypropylene at high cooling rates. 12th ESAFORM Conference on Material Forming, Apr 2009, Enschede, Netherlands. pp.Pages 857-860, 10.1007/s12289-009-0631-5 . hal-00509429

\section{HAL Id: hal-00509429}

https://hal-mines-paristech.archives-ouvertes.fr/hal-00509429

Submitted on 12 Aug 2010

HAL is a multi-disciplinary open access archive for the deposit and dissemination of scientific research documents, whether they are published or not. The documents may come from teaching and research institutions in France or abroad, or from public or private research centers.
L'archive ouverte pluridisciplinaire HAL, est destinée au dépôt et à la diffusion de documents scientifiques de niveau recherche, publiés ou non, émanant des établissements d'enseignement et de recherche français ou étrangers, des laboratoires publics ou privés. 


\title{
CRYSTALLIZATION OF POLYPROPYLENE AT HIGH COOLING RATES
}

\author{
J.M. Haudin ${ }^{1 *}$, S.A.E. Boyer ${ }^{1 * *}$ \\ ${ }^{1}$ MINES Paris-Tech, CEMEF - Centre de Mise en Forme des Matériaux, CNRS UMR 7635, 1 Rue \\ Claude Daunesse, 06904 Sophia Antipolis Cedex, France
}

\begin{abstract}
In the context of polymer crystallization under high and constant cooling rates, a new survey is presented. The growth kinetics of spherulites from the molten state and the corresponding temperature of crystallization under similar cooling rates are considered. An industrial grade of isotactic polypropylene (iPP) is investigated in the range of slow (from 1 to $10^{\circ} \mathrm{C} / \mathrm{min}$ ), of relatively moderate (from 30 to $500^{\circ} \mathrm{C} / \mathrm{min}$ ) and of high (from 500 to $1600^{\circ}$ $\mathrm{C} / \mathrm{min}$ ) constant cooling rates. The growth kinetics for the $\alpha$-modification of iPP versus the temperature of crystallization is in agreement with the data published by Janeschitz-Kriegl [Macromolecules, 2006]. To understand the growth mechanism, the Hoffman equation is used and then extrapolated to lower temperatures of crystallization. Finally, to describe the non-isothermal crystallization, the equation of Ozawa is used. The benefits to use the spherulitic growth kinetics to extrapolate data from alternative methods, i.e., differential scanning calorimetry (DSC), are briefly discussed.
\end{abstract}

KEYWORDS: Industrial Processes, Liquid to Solid Transition, Isotactic Polypropylene, Kinetics, Polarized Optical Microscopy, Differential Scanning Calorimetry

\section{INTRODUCTION}

Liquid to solid transition of polymers at high cooling rates has to be well documented in order to be modeled and implemented in simulation codes describing the relationships between polymers and thermo-mechanical conditions encountered in processing conditions, like in injection-molding [1]. If we focus the work on thermal effects, the attractive point supposes to adopt cooling procedures comparable with those undergone by the polymer during processing and to reproduce them. An approach is to consider that crystallization mechanism understanding requires a minimum of information on growth kinetics at the spherulitic scale.

The present study is concerned with the crystallization behavior under high and constant cooling rates from 50 to $1600^{\circ} \mathrm{C} / \mathrm{min}$. For that purpose, a novel approach involving a new home-made set-up named «Polymer High Cooling - Optics » is introduced. The optical setup recently developed in our laboratory [2] has been revisited [3]. It allows us to determine the temperature of crystallization together with the spherulitic growth kinetics versus constant cooling rates. The extraction of the parameters is done by classical methods. A comparison with literature data is established. In order to model the non-isothermal crystallization from Ozawa equation, the benefits to use the spherulitic growth kinetics to extrapolate data from an additional method, i.e., DSC, are briefly discussed.

\section{OVERVIEW OF HIGH COOLING POLYMER CRYSTALLIZATION}

\subsection{POLYMER CRYSTALLIZATION THEORIES}

At the spherulitic scale and in quiescent conditions, polymer crystallization is described by means of nucleation and growth, or in a more global manner by the overall kinetics. The nucleation and growth steps are used for the overall description, e.g., in the approaches developed by Kolmogoroff, Avrami and Evans [1]. These approaches are generally used in isothermal conditions. Description of non-isothermal crystallization uses Ozawa equation [4], which is derived from Evans theory [5]. Attempts to describe growth mechanism were several times proposed by Hoffman and Lauritzen [6]. Their model still remains the only one satisfactory.

\subsection{SPECIFIC EXPERIMENTAL SET-UPS}

Techniques for measuring rates of crystallization have been the subject of reviews. The phase change has to be monitored in real-time from a direct observation of the structure development or from an effect resulting from the structure development, e.g., a thermal effect. To observe the structure development under high cooling rates, special hot stages must be constructed and appropriate methods proposed. For instance, Ding \& Spruiell [7] proposed a light-depolarizing microscopy

\footnotetext{
* Corresponding author: MINES ParisTech, Tel :33 49395 (75 03)*, (75 65)**, Fax: 33492389752

Email address: Jean-Marc.HAUDIN@mines-paristech.fr *

Email address: Severine.BOYER@mines-paristech.fr **
} 
(LDM) technique for studying crystallization of a polymer film, $150 \mu \mathrm{m}$ in thickness, at average cooling rates up to $5000^{\circ} \mathrm{C} / \mathrm{min}$. Brucato, Piccarolo and Titomanlio proposed a general experimental route involving the recording of the thermal history of a thin polymer film, 50-100 $\mu \mathrm{m}$ in thickness, and the detection of depolarized light intensities in the course of polymer crystallization [8]. Very high cooling rates up to $2000^{\circ}$ $\mathrm{C} / \mathrm{s}$ were attained.

The structure development can be characterized from thermal analysis by means of Differential Scanning Calorimetry (DSC). For instance, Pijpers et al. have shown that DSC can be used at rates up to $500 \mathrm{~K} / \mathrm{min}$ [9] to study the rate-dependent phenomena in real-time; but no more investigation were done. To attain higher cooling rates, an ultra-fast thin film calorimeter, or nanocalorimeter, was proposed by Adamovsky \& Schick [1013]. Rates of more than $1000 \mathrm{~K} / \mathrm{s}$ can be attained, both in cooling and in heating modes.

\section{EXPERIMENTAL SECTION}

\subsection{MATERIAL}

One isotactic polypropylene (iPP) grade for injection molding was chosen because it produces spherulites easily observable by optical microscopy. The commercial reference is 3250 MR1 (ATOFINA Company, France) (now named PPH9081, TOTAL PETROCHEMICALS) with a weight average molecular weight $M w$ of $213000 \mathrm{~g} / \mathrm{mol}$ and a polydispersity $P D$ of 5. The sample is a film of $50 \mu \mathrm{m}$ in thickness.

\subsection{NEW "POLYMER HIGH COOLING" SET-UP}

A newly developed and revisited hot stage was used. The technique combines a special designed optically transparent hot-stage together with a polarizing optical microscopy system.

\subsubsection{Polarizing Optical Microscopy}

The one-line monitoring of the structural evolution uses optical light microscopy, with a LEICA-DMRX microscope (Leica-Microsystèmes, France). Crystalline entities are observed between crossed polarizers, $+/-90^{\circ}$ rotated. A digital CMOS camera is used for the one-line monitoring.

\subsubsection{Thermal control of the polymer sample}

With the choice of a transparent hot-stage as a framework, in-situ observation of the crystallization is achieved. The polymer is placed between a pair of thin microscope glasses. It is heated and cooled down by a gaseous flowing supplied at a constant temperature. The thermo-sensor is inserted into the polymer film to eliminate lag time between the polymer and the heat transfer medium. Careful attention was paid to locate the thermo-sensor very near to the optical observation area. These positions ensure a realistic polymer temperature. Relatively stable temperature conditions for the sample were provided as illustrated in Figure 1. The thermal protocol was: $i$ ) rapidly heating the sample to about 215
${ }^{\circ} \mathrm{C}$, ii) about $90 \mathrm{~s}$ holding at $215^{\circ} \mathrm{C}$ to erase the thermal history and to avoid its degradation, enabling thus the processing of the sample for many cycles, iii) cooling at various cooling rates $\dot{T}$.

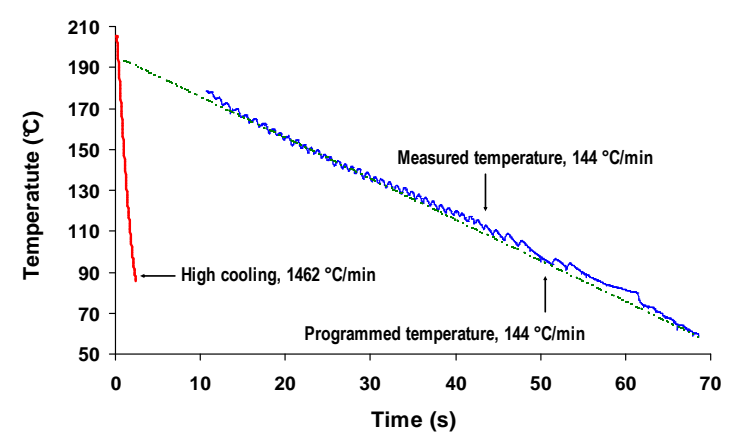

Figure 1: Temperature-time evolution of iPP sample at a constant programmed cooling rate of $144{ }^{\circ} \mathrm{C} / \mathrm{min}$ and at a high cooling rate of $1462{ }^{\circ} \mathrm{C} / \mathrm{min}$ using the mode 'all-ornone'.

\subsubsection{Sequence and acquisition}

A Labview program was especially written in order to program the cooling rates, the temperature profile of the sample and the monitoring of optical observation. Two cooling modes are possible: a proportional one using a rotating throttle to control constant cooling rates at relatively moderate values (from 30 to $500{ }^{\circ} \mathrm{C} / \mathrm{min}$ ) and an 'all-or-none' one using an 'open-close' valve for cold flux to attain high cooling rates (from 500 to $1600^{\circ}$ $\mathrm{C} / \mathrm{min}$ ).

\section{RESULTS \& DISCUSSIONS}

Different films of iPP were investigated in a broad range of slow (from 3 to $10^{\circ} \mathrm{C} / \mathrm{min}$ ) with a conventional Mettler hot-stage, of relatively moderate (from 30 to 500 ${ }^{\circ} \mathrm{C} / \mathrm{min}$ ) and of high (from 500 to $1600{ }^{\circ} \mathrm{C} / \mathrm{min}$ ) cooling rates. Figure 2 shows the structure development investigated with the newly developed «Polymer High Cooling - Optics » set-up. The two cooling processes are illustrated, i.e., relatively moderate rates as shown at 144 ${ }^{\circ} \mathrm{C} / \mathrm{min}$ (Figure 2a) and high cooling rates as shown at $1462{ }^{\circ} \mathrm{C} / \mathrm{min}$ (Figure 2b).
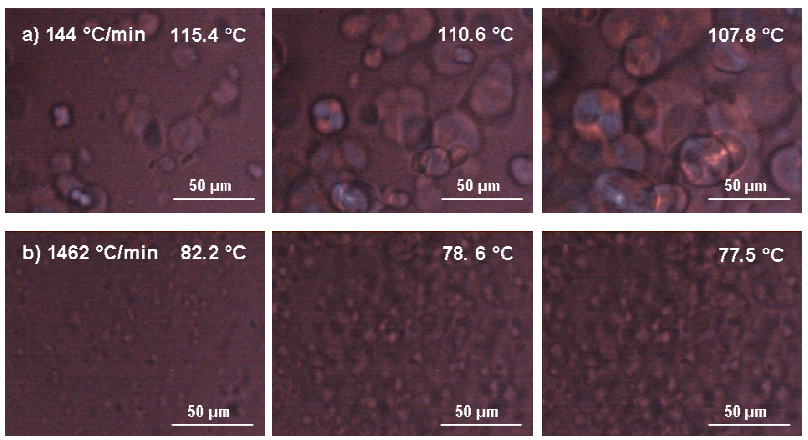

Figure 2: Development of iPP spherulites at a constant cooling rate a) $144{ }^{\circ} \mathrm{C} / \mathrm{min}$ and b) $1462{ }^{\circ} \mathrm{C} / \mathrm{min}$. Two different iPP films were used. 
As expected at first glance, when the cooling rate increases, the crystallization occurs at lower temperature, about $30^{\circ} \mathrm{C}$ lower at $1462{ }^{\circ} \mathrm{C} / \mathrm{min}$ compared to $144^{\circ}$ $\mathrm{C} / \mathrm{min}$. The nuclei are randomly distributed over the area of observation; their density rapidly increases with time and with cooling rate. The size distribution of crystallized entities decreases with increasing cooling rates. The growing entities have a radial growth direction. During crystallization, no drastic birefringent contrast was observed, $\alpha$-modification is predominant.

With the present « Polymer High Cooling - Optics » setup, it is possible to discern and to follow one-line the spherulitic crystallization at high cooling rate up to 1600 ${ }^{\circ} \mathrm{C} / \mathrm{min}$.

Figure 3 plots the growth kinetics $G$ against the temperature of crystallization $T_{c}$. The newly obtained spherulite growth rates are plotted with the ones collected by Janeschitz-Kriegl [14].

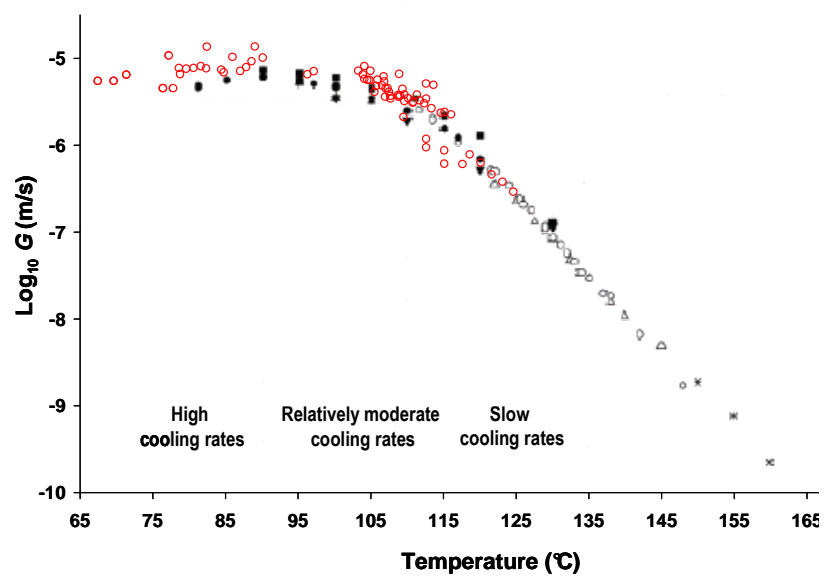

Figure 3: Compilation of iPP $\alpha$-spherulites growth rates versus the crystallization temperatures. Red open circles are from the present investigation at high and relatively moderate cooling rates (from 50 to $1600 \mathrm{C} / \mathrm{min}$ ); da ta at slow cooling rates are from an investigation using a conventional Mettler hot-stage (at 3 and $10^{\circ} \mathrm{C} / \mathrm{min}$ ). Open symbols are from Lovinger et al., 1977 (rhombs), Padden \& Keith, 1959 (circles), Von Falkai, 1960 (triangles), Olley \& Bassett, 1989 (stars). Closed symbols are from: $\beta$-transcrystallization, Ratajski, 1993 (closed squares), microtome slices, Ratajski \& Janeschitz-Kriegl, 1996 (closed circles), DSC, Paulik, 1993 (closed triangles pointed upward) and Ratajski \& Janeschitz-Kriegl, 1996 (closed diamonds), incubation times, Ratajski \& Janeschitz-Kriegl, 1996 (closed triangles pointed downward). Literature data are from reference [14].

The growth kinetics $G$ follows the bell-shaped dependence with the temperature of crystallization. It is remarkable to observe that with only one method, the growth kinetics $G$ in the range $65-125^{\circ} \mathrm{C}$ can be well investigated. The information collected from the literature data do not show a so broad range of investigated $T_{c}$ resulting from one study. At relatively moderate cooling rates, the growth kinetics $G$ increases with undercooling up to around $90^{\circ} \mathrm{C}$. For high cooling rates, $T_{c}$ attains $65^{\circ} \mathrm{C}$, the growth kinetics $G$ seems to gradually decrease. This tendency is not very accentuated probably due to the limitation of use of the set-up.

According to the Hoffman and Lauritzen theory [6], as written in equation (1), Figure 4 plots the growth kinetics $G$ as a function of the temperature of crystallization $T_{c}$ and the degree of undercooling $\Delta T$ with $\Delta T=T_{m}^{0}-T_{c}$.

$T_{m}^{0}$ is the equilibrium melting temperature.

$$
\ln G+\frac{U^{*}}{R\left(T_{c}-T_{\infty}\right)}=\ln G_{0}-\frac{K_{g}}{T_{c}(\Delta T)}
$$

$U^{*}$ and $T_{\infty}$ are the Vogel-Fulcher-Tamman-Hesse (VFTH) parameters. $U^{*}$ is the activation energy for the transportation of segments of molecules across the melt/solid surface boundary and is usually given by a universal value of $1500 \mathrm{cal} / \mathrm{mol} . T_{\infty}$ is the temperature where the molecular motion ceases with $T_{\infty}=T_{g}-30 . R$ is the universal gas constant. $G_{o}$ is a pre-exponential term. $K_{g}$ is the nucleation parameter. It was estimated to $8.0110^{5} \mathrm{~K}^{2}$ from relatively moderate constant cooling rate investigation and was in agreement with literature data [15].

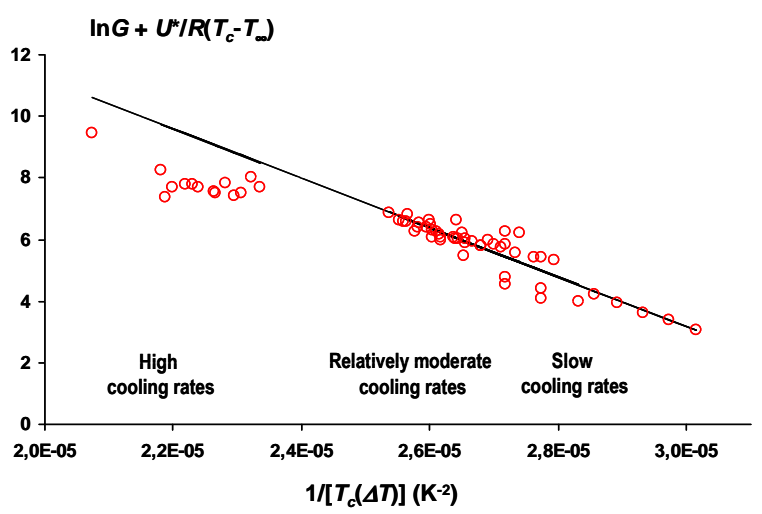

Figure 4: Spherulite growth rates of iPP versus the crystallization temperatures $T_{c}$ based on the Lauritzen \& Hoffman secondary nucleation theory with $T_{m}^{0}=208^{\circ} \mathrm{C}$ and $T_{g}=-21^{\circ} \mathrm{C}$.

From now, the benefits are to use the growth kinetics $G$ versus the temperature of crystallization $T_{c}$ curve to extrapolate data from alternative differential scanning calorimetry methods. Cooling rates extended from 1 to $500^{\circ} \mathrm{C} / \mathrm{min}$ were examined. The calculated overall kinetics allows us to experimentally extend the Ozawa model to $55^{\circ} \mathrm{C}$, a temperature which approaches the process temperatures.

The Ozawa model can then be extrapolated theoretically at low temperature thanks to the estimate of the initial number of nuclei per unit volume $N_{0}$ from the DSC experiments and thanks to Hoffman \& Lauritzen theory. 


\section{CONCLUSIONS}

The major task of the present paper is to tackle the polymer growth kinetics under high cooling rates. A newly developed and revisited set-up, named « Polymer High Cooling - Optics », first generation in date, was employed. It allows us to simultaneously follow the polymer temperature and the radial growth of spherulites during cooling rates up to $1600{ }^{\circ} \mathrm{C} / \mathrm{min}$. The spherulitic growth rate follows the typical bell-shaped dependence with the temperature of crystallization in a broad range.

The benefits are to use the newly plotted growth kinetics $G$ versus the temperature of crystallization $T_{c}$ to extrapolate the Ozawa model constructed for the overall kinetics of crystallization.

At present, the control of the kinetics of crystallization from the melt at higher cooling rates, higher than $1600^{\circ}$ $\mathrm{C} / \mathrm{min}$, would be of interest especially to conclude or not on the bell-shape of growth kinetics. It would be needed to think about a second generation of « Polymer High Cooling - Optics » set-up.

Nevertheless, additional analysis of the acquired data, i.e., optimization of the growth kinetics, estimation of the density of nuclei when it is possible, would be used for implementation into new crystallization models and then into numerical simulation.

\section{ACKNOWLEDGEMENT}

Acknowledgements are presented to the CEMEF technical team for their support during the revisit of the set-up. Perkin-Elmer company is also acknowledged for specific DSC experiments

\section{REFERENCES}

[1] J.-M. Haudin, J.-L. Chenot. Numerical and physical modelling of polymer crystallization. Intern. Polym. Process., 19: 267-274, 2004.

[2] E. Da Passano, B. Monasse, MINES ParisTech report, September 2006

[3] M. Brisebourg, S.A.E. Boyer, MINES ParisTech report, September 2008

[4] T. Ozawa. Kinetics of non-isothermal crystallization. Polymer, 12: 150-158, 1971.

[5] U.R. Evans. The laws of expanding circles and spheres in relation to the lateral growth of surface films and the grain-size of metals. Trans. Faraday Soc., 41:365-375, 1945.

[6] J.I. Lauritzen Jr., J.D. Hoffman. Theory of formation of polymer crystals with folded chains in dilute solution. J. Res. Natl. Bur. Stand. A, 64:73$102,1960$.

[7] Z. Ding, J.E. Spruiell. An experimental method for studying nonisothermal crystallization of polymers at very high cooling rates. J. Polym. Sci. B. Polym. Phys., 34:2783-2804, 1996.
[8] G. Lamberti, F. De Santis, V. Brucato, G. Titomanlio. Modeling the interactions between light and crystallization polymer during fast cooling. Appl. Phys. A, 78:895-901, 2004.

[9] T.F.J. Pijpers, V.B.F. Mathot, B. Goderis, R.L. Scherrenberg, E.W. Van Der Vegte. High-speed calorimetry for the study of the kinetics of (de)vitrification, crystallization, and melting of macromolecules. Macromolecules, 35:3601-3613, 2002.

[10] S.A. Adamovsky, C. Schick. Ultra-fast isothermal calorimetry using thin film sensors. Thermochim. Acta, 415:1-7, 2004.

[11] S.A. Adamovsky, A.A. Minakov, C. Schick. Thermochim. Acta, 403:55-63, 2003.

[12] A.A. Minakov, S.A. Adamovsky, C. Schick. Thermochim. Acta, 432:177-185, 2005.

[13] A. Gradys, P. Sajkiewicz, A.A. Minakov, S. Adamovsky, C. Schick, T. Hashimoto. K. Saijo, Crystallization of polypropylene at various cooling rates. Mater. Sci. Eng., A413-414: 442-446 (2005).

[14] H. Janeschitz-Kriegl. Phases of flow-induced crystallization of i-PP: how remote pieces of the puzzle appear to fit. Macromolecules, 39:44484454, 2006.

[15] J. Xu, S. Srinivas, H. Marand, P. Agarwal. Equilibrium melting and undercooling dependence of the spherulitic growth rate of isotactic polypropylene. Macromolecules, 31:8230-8242, 1998. 